\title{
Nurses' Perception toward the Effectiveness of Using Risk Fall Prevention Protocol of Hospitalized Patients.
}

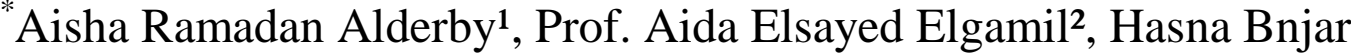 \\ ${ }^{1}$ (Faculty of nursing, Master Student, Medical Surgical department, KingAbdul Aziz University, Najran \\ University, Saudi Arabia) \\ ${ }^{2}$ (Faculty of nursing, Alexandria University, Egypt) \\ ${ }^{3}$ (Assistant Professor of Leadership and Management in Nursing, Faculty of nursing, King Abdul \\ Aziz University, Saudi Arabia) \\ Corresponding Author: Aisha Ramadan Alderby
}

\begin{abstract}
Falls are a widespread concern in hospitals setting. Fall prevention is a very pressing hospital risk management and patient safety challenge due to many of in-patient admitted for mobility problems, falls, or injury from falls making.The aim of the study is to assess nurses' perception of the effectiveness of using fall prevention protocol of risk fall hospitalized patients. The study is quantitative descriptive cross-sectional design. Sample: A convenience sample of 204 nurse from KAUH. Data collection was used in this study was "Assessment of the injurious fall risk factors and fall prevention intervention questionnaire". Result:Overall, Nurses coming from the general Units perceived significantly higher frequently perceived fall-risks, greater Perceived nursing intervention. Conclusion: It is concluded that nurses' perception towards frequently preventable risk factors and their frequently intervention to prevent those factors has positive strong relationship and got the highest scored mean. Recommendation: all nurses should be motivated and encouraged to attend regular continuous education of programs every six months for risk fall and prevention measures.
\end{abstract}

Keywords: nurses perception, fall prevention, risk factors and hospitalized patients.

\section{Introduction}

Falls are a widespread concern in hospitals settings (Oliver et al., 2010). Fall "is an unexpected event in which an individual comes to the ground floor or lower level" (Martin, 2011). Preventing and managing inpatients falls in healthcare setting is a great challenge in and medical surgical nursing(MacCulloch et al., 2007).Inpatient falls are related with long hospital stay and result in increased costs for health facilities (Callis, 2016).Patient fall are perceived as an indicator which could be improved by applying safety strategies and interventions by nurses (El Enein et al., 2012). Many studies investigaterisk factors for fall (Eto, 2001; MacCulloch et al., 2007; Wayland et al., 2010; Tung and Newman, 2014).Risk factors for fall are classified into Intrinsic factors and Extrinsic factors.First, Intrinsic factors: are those factors that originated from patients themselves related to their body systems which put them in risk for fall. These internal factors of individuals and populations such as age, history of falls, vision impairment, balance disorders, impaired mobility, lower extremity weakness, poor grip strength). Second,Extrinsic factors: are external factors that face patients and effect their safety such as medication (e.g. Benzodiazepines, Antipsychotics, Antidepressants, Sedatives, Opiates), or environmental aspects (e.g. slippery walking surfaces). The incidence of inpatients falls in the general medical and surgical in king Abdul Aziz university hospital showed to be high as much as $2.4 \%$ (27 falls) in 3 months only (Al Thadli, 2009).

1.1 Aim of the study :

Is toassess nurses' perception toward the effectiveness of using risk fall prevention protocol of hospitalized patients.

\subsection{Research Questions:}

Q1: What are the nurses' perception of falls prevention protocols for risk fall hospitalized patients?

Q2: What are the most percieved items of frequently risk of fallsandeffectiveness of nursing interventions?

\subsection{Research Design}

\section{Methods}

A quantitative descriptive cross-sectional design was used to answer the researcher question. 


\subsection{Study Setting and Sampling:}

The sample for the research was recruited from King Abdul-Aziz university hospital (KAUH) .It is educational hospital, in Jeddah, Kingdom of Saudi Arabia. This study was conducted in inpatient Acute Units (Medical, Surgical and day care male or female) and Critical Units (Intensive Care, Emergency Department, dialysis and cardiac units). A convenience sample of 204 nurses. All nurses have experience more than 12 months in the current workplace and working in morning and night shift.

\subsection{Ethical Consideration:}

Approval was obtained from the administration of the Nursing College, KAUH to conduct the study.

Participation in the study was voluntary, and anonymous.

\subsection{Tool:}

The data collection tool used "Assessment of the injurious fall risk factors and fall prevention intervention questionnaire". It was developed by the (Tzeng, 2013) and modified by researcher after taking permission from the author. It consist of three parts

Part I: Socio demographic data includes primary working shift, department, sex, and years of experience, educational level and receiving fall prevention program).

\section{Part II: The injurious fall risk factors questionnaire.}

It Includes 44 potential factors e.g. (impaired balance, cognitive impairment, comorbidity of disease, medication and environment hazards) that may lead to an injurious fall in adult inpatients (aged 21 years or older). Nurses were asked to rate these factors using 5-point likert scale, based on their experience in their current unit.

\section{Part III: The Fall prevention intervention questionnaire.}

It Consists of 40 interventions for preventing injurious falls in adult inpatients e.g. (assess environment as needed, keep floor clean and reduce clutter). Based on the nurse's experience in their current unit they were asked to rate these factors using 5 points likert scale. Higher scores in fall prevention intervention questionnaire indication higher-ranking order.

\section{5 validity and Reliability}

Questionnaire was reviewed by five experts in the field of medical surgical nursing department, faculty of nursing, KAU.Questionnaires of the current study was tested by Cronbach's alpha and it is found to be (alpha= 0.99 and 0.97 ) respectively for the two main concept.

\subsection{Statistical analysis}

Statistical analysis was performed using statistical package for social science (SPSS) version 21 for windows.Descriptive statistics was used to calculate means and frequencies, Summative analysis was used to compute a mean score for the two main concepts namely "Perceived frequently risk of falls" and the "frequently nursing interventions for fall preventions". Means were ranked ascending and we reported the top 10 perceived the two main concept.

\section{Results}

Table 1 shows that $86.3 \%$ of nurses were Female and the rest were male. More than one third of nurses had one to five years of experience $43.6 \%$. Regarding nurses education more than half of participant nurses $52.5 \%$ had diploma in nursing . Finally, as regards to attendances of Fall prevention training program, more than two thirds of participant nurses $62.7 \%$ were attend last fall prevention training program <6months ago .

\begin{tabular}{|c|c|c|}
\hline $\begin{array}{l}\text { Socio demographic } \\
\text { characteristic }\end{array}$ & NO & $\%$ \\
\hline Gender: & & \\
\hline Female & 176 & 86.3 \\
\hline Male & 28 & 13.7 \\
\hline Years of experience & & \\
\hline $1->5$ years & 89 & 43.6 \\
\hline $1->5$ years & 75 & 36.8 \\
\hline $1->5$ years & 27 & 13.2 \\
\hline $1->5$ years & 13 & 6.4 \\
\hline Nursing education & & \\
\hline Diploma & 107 & 52.5 \\
\hline BSN & 97 & 47.5 \\
\hline Last fall prevention program & & \\
\hline$<6$ months ago & 128 & 62.7 \\
\hline$>1$ years & 50 & 24.5 \\
\hline No training & 26 & 12.7 \\
\hline
\end{tabular}

Table2 illustrate the top ten perceived most frequent falling risks factors were patients with (impaired balance, next were patients with altered mobility, then patients subject to slippery floors and confused patients, 
then patients with Alzheimer's disease and those with disorientation, next was those patients who received Antiepileptic's and /anti-convulsion drugs, followed by those patients who are visually impaired. The next ranks of Observable were given to lack of handrails in bathrooms and finally the patients who are unable to follow safety instructions).

Table (2) ranking order of top 10 perceived items of patients and environmental risk factors of fall in the hospital as perceived by nurses.

\begin{tabular}{|l|c|c|}
\hline Patient related risk factors & Mean \pm SD & Ranking order \\
\hline Impaired balance & $3.72 \pm 1.4$ & 1 \\
\hline Altered or limited mobility/gait problems & $3.71 \pm 1.3$ & 2 \\
\hline Confusion. & $3.62 \pm 1.3$ & 4 \\
\hline Alzheimer's disease. & $3.59 \pm 1.4$ & 5 \\
\hline Disorientation. & $3.58 \pm 1.4$ & 6 \\
\hline Antiepileptic's/anticonvulsants & $3.54 \pm 1.4$ & 7 \\
\hline Visual impairment. & $3.54 \pm 1.4$ & 8 \\
\hline Environmental hazards risk factors & & \\
\hline Slippery or wet floor surfaces & $3.62 \pm 1.4$ & 3 \\
\hline Lack of handrails in patient room bathroom. & $3.53 \pm 1.4$ & 9 \\
\hline Inability to follow safety instructions. & $3.51 \pm 1.4$ & 10 \\
\hline
\end{tabular}

Table3present the distribution of the effective Nursing Interventions to Prevent Injurious Falls in hospital. Regarding to the Top 10: (Keeping Hospital Brakes On, followed by keeping hospital floors dry, clean and crack-free, then maintaining patient call lights within patient's reach mean. Next was keeping beds in lowest position, and adjusting lighting to daily activities of patients, and equally for each of instructing patients to raise themselves slowly from the beds and administering the fall-risk assessment scales at admission time. The eighth rank was reorienting the patients about the place and space when required, then All clinical and non-clinical staff understand the institution's policies and procedures in place for the prevention of falls and finally the then teaching patients on how to use call lights).

Table (3) Present ranking order of top 10 effective nursing interventions to prevent injurious falls in hospital.

\begin{tabular}{|l|l|l|}
\hline Nursing intervention & Mean \pm SD & Ranking order \\
\hline Keep hospital bed brakes locked. & $4.45 \pm 0.97$ & 1 \\
\hline Keep floor surfaces clean and dry. & $4.43 \pm 0.92$ & 2 \\
\hline Maintain call light within reach. & $4.42 \pm 0.94$ & 3 \\
\hline Place patient bed in low position & $4.37 \pm 1.04$ & 4 \\
\hline Adjust the lights for activities of daily living & $4.34 \pm 1.08$ & 5 \\
\hline Instruct patient to rise slowly, & $4.29 \pm 0.98$ & 6 \\
\hline at admission Complete a fall risk assessment & $4.29 \pm 0.98$ & 7 \\
\hline Reorient patient about the environment & $4.28 \pm 1.06$ & 8 \\
\hline $\begin{array}{l}\text { All clinical and non-clinical staff understand the } \\
\text { institution's policies and procedures in place for the } \\
\text { prevention of falls. }\end{array}$ & $4.27 \pm 1.00$ & 9 \\
\hline Have patient demonstrate call light use & $4.26 \pm 1.02$ & 9 \\
\hline
\end{tabular}

Table four illustrate the Nurses perceived 3.2 out maximum five possibly frequent falls that mean fall risk categories were perceived on average between sometimes to often occurring. Regarding to theNurse Measures were perceived as often helpful in most occasions on average mean $=4.1$ out of five. The majority of nurses answer (4.7) or less in the effectiveness of the nursing interventions and the perceived risk factor answer (3.9) or less.

TABLE 4: Illustration of mean and SD of nurses perception of the injurious risk factors and nursing intervention as perceived by nurses.

\begin{tabular}{|l|c|c|c|}
\hline \multicolumn{1}{|c|}{$\begin{array}{c}\text { Nurses perception } \\
\text { concepts }\end{array}$} & Mean \pm SD & $\begin{array}{l}\text { Minority of (25\%) } \\
\text { nurses perception }\end{array}$ & $\begin{array}{c}\text { Majority of (75\%) } \\
\text { nurses perception }\end{array}$ \\
\hline $\begin{array}{l}\text { 1. perceived Risk factors } \\
\text { of Fall. }\end{array}$ & $3.22 \pm 1$ & 2.6 & 3.9 \\
\hline $\begin{array}{l}\text { 2. Effective Nursing } \\
\text { Interventions to Prevent } \\
\text { Injurious Falls. }\end{array}$ & $4.1 \pm 0.8$ & 3.8 & 4.7 \\
\hline
\end{tabular}

Table 5 revealed that the T-test showed that Nurses working at the general Units perceived significantly higher $($ mean $=3.2 \pm 0.95)$ perceived risk of falls than those working in the Critical Units (mean= $2.9 \pm 0.99), \mathrm{p}=0.002$, $\mathrm{t}$-value $=-3.12$. However, Nurses working in Critical Units and those working within the general Units were not statistically different on their perceptions of the effective nurses intervention at 
preventing the risks of fall, $\mathrm{p}=0.371$, denoting they all share a consensus on the effective of nursing intervention at preventing risks of falling down for an hospitalized patient.

TABLE 5: illustrate Most Frequently Hospital Practiced Interventions within critical and acute Units.

\begin{tabular}{|l|c|c|c|c|}
\hline $\begin{array}{l}\text { Nurse's Perceptions } \\
\text { four concept }\end{array}$ & $\begin{array}{c}\text { Critical Units, } \mathbf{n}=\mathbf{9 5} \\
\text { Mean } \pm \text { SD }\end{array}$ & $\begin{array}{c}\text { general unit, } \mathbf{n = 1 0 9} \\
\text { Mean } \pm \text { SD }\end{array}$ & $\begin{array}{c}\text { t-value } \\
(\mathbf{D F}=\mathbf{2 0 2})\end{array}$ & P \\
\hline Perceived risk of Fall & $2.9 \pm 0.99$ & $3.2 \pm 0.95$ & -3.120 & 0.002 \\
\hline $\begin{array}{l}\text { effective Nursing } \\
\text { Interventions }\end{array}$ & $4.1 \pm 0.69$ & $4.2 \pm 0.85$ & -0.897 & 0.371 \\
\hline
\end{tabular}

Table 6 show significant relationship with the Nurses perception of the frequency of perceived patientfalls. Nurses trained within the past six months (mean=3.41 \pm 0.9 ) perceived significantly higher frequency of fallers than those who had No training on fall prevention (mean=2.88 \pm 1.1 ), $\mathrm{p}=0.04$ everything else is kept constant. Conversely those Nurses trained one year ago or more were Not too different with their perceptions on frequency of perceived falling patients than those who had No training, $p=0.661$.

TABLE 6: Multivariate Linear Regression Explaining the Joint \& Individual Relationship between Nurse Characteristics with the mean perceived risk of falls.

\begin{tabular}{|l|l|l|}
\hline Nurses characteristics & Mean \pm SD & P value \\
\hline (Constant) & $\mathbf{3 . 2 2} \pm \mathbf{1}$ & $\mathbf{. 0 0 1} * *$ \\
\hline Years of experience & & \\
$1>5$ & $3.24 \pm 1$ & .693 \\
$6>10$ & $3.11 \pm 0.9$ & .475 \\
$11>15$ & $3.28 \pm 1.2$ & .750 \\
$16>20$ & $3.41 \pm 1$ & .510 \\
\hline Nurse Education: & & \\
BSN. & $3.30 \pm 0.9$ & .166 \\
Diploma & $3.16 \pm 1.1$ & -.166 \\
\hline Trained on Fall Prevention> 1 & & \\
year. & $2.84 \pm 1$ & .661 \\
Trained on Fall Prevention 6 & $\mathbf{3 . 4 1} \pm \mathbf{0 . 9}$ & $\mathbf{. 0 4 1} *$ \\
months & $\mathbf{2 . 8 8} \pm \mathbf{1}$ & $\mathbf{. 6 5 1}$ \\
No training & & \\
\hline
\end{tabular}

Figure 1: Correlation between perceived risk of fall and frequently nursing intervention.

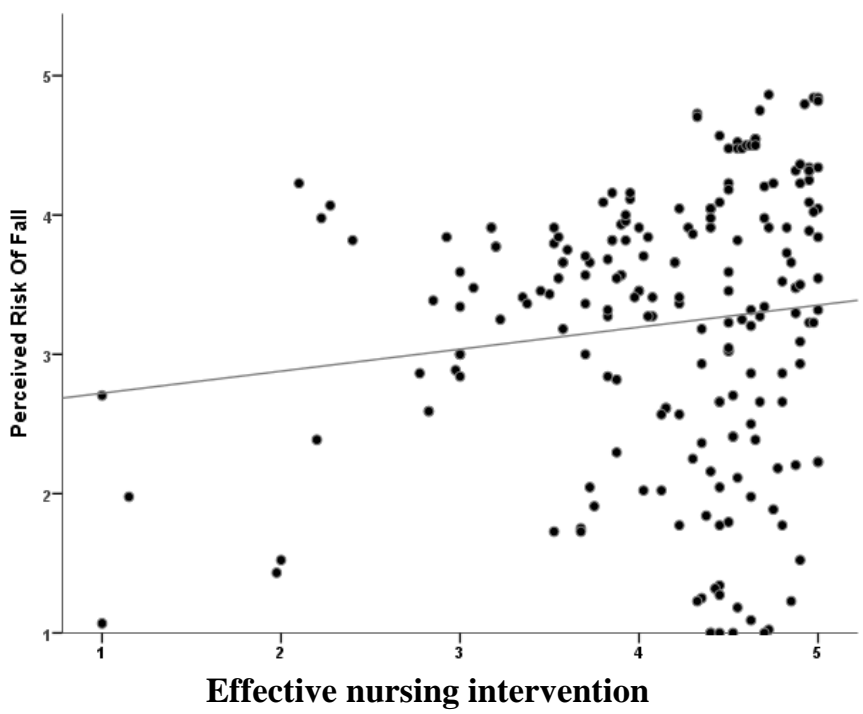

We can see in the scatter plots that as nurses perceived (perceived risk factors ) tends to increase, their perception on (frequently nursing intervention) also tended to incrementally increase, denoting an association between two concepts $r=.125, p=.05$.

\section{Discussion}

This cross-sectional hospital-based nurse survey was conducted in a university hospital. The aim of this study is toassess nurses' perception of the effectiveness of using fall prevention protocol of risk fall hospitalized patients. A total of 204 of female and male nurses were recruited to participate in the study. Results the study 
are discussed here. In the KAUH utilizes a fall prevention policy as standard of care of all nursing staff to minimize inpatient falls. This fall prevention policy allows the nursing staff to assess inpatients on admission and this is recorded using a fall risk assessment tool and kept in the patients' files.

The following risk factors like impaired balance, altered or limited mobility, wet floor, confusion, Alzheimer disease, disorientation, anticonvulsants, visual impairment, lack of handrails in patients' rooms and bathrooms, inability to follow safety instructions, improper use of side rails and antipsychotics occurred more frequently to those nurses, which in turn, they perceived certain nursing interventions might help much in preventing injurious falls of their patients as a result of the mentioned risk factors.

In this study, impaired balance considered as a risk factor of fall patient, scored the highest mean value that had been identified by the nurses' participants and perceived as the most perceived risk factor for inpatients' falls. Impaired balance and instable gait by patients' are top risk factors among the common top ten most perceived risk factors to cause inpatients' falls as perceived by nurses' participants. The same perception was identified by Amir, et al 2016, concluded that high parathyroid hormone for patients under hemodialysis is causing imbalance gait that put those patients at high risk for fall.

Medications are playing important role in putting patients at risk of falls. This is especially in fragile patients, like the elderly inpatients when using many different type of medication, such as antihypertensive (Ziere G, et al 2006). Sleep inducing drugs,cough formula, and antiplatelet(Kaysar \& Jimmy, 2009), especially when used with other medications for treating certain conditions during the older patients' stay, this may be due to the effect on the function of the muscular, skeletal and nervous system. Nurses in this study do not perceive medication as high risk factor for fall in their hospital, which might be due to either less elderly and mentally ill patients in high density of admitted cases to the hospital. Another study Contradicting to this finding, reported certain medications, such as, sleep-induced drugs, cough formula and antiplatelet is considered an individual and internal risk factor for fall in majority of those elderly inpatients as determined by Al Saif et al 2012, and Kaysar et al 2009.Others, as adult inpatients are also identified prone to fall while they use different type of medications, for example, chemotherapy for patients with Myeloid Leukemia (Kristin, et al 2011).

Nurses' participants in this study mentioned the most frequent top ten nursing actions that they are practicing in a form of multidisciplinary interventions strategy as important effective interventions for falls prevention. These interventions are practiced by nurses' participants and showed to be effective in minimizing and preventing patients' falls. Those multi-actions practiced by nurses are known to be multidisciplinary fall prevention interventions strategies. They are collaborative actions planned in order to be implemented to minimize and avoid fall of inpatients (AlSenany 2010, Elenein et al, 2012, Bunn et al, 2014, Kaysar et al 2009, Gutierrez \& Smith 2008, Sandra et al 2012). From the top ten actions practiced by nurses' participants to be effective are locking bed-brakes, clean and dry floor, call-light, low-bed position, lighting the room during day, these are the first four actions with the highest mean. Filling fall risk assessment form at admission also shown to be an effective action. Nurses' instruction to their patients regarding rising slowly from bed and to press button for call are proven to be effective also. Giving full orientation about the patient's environment promote their safety too. William, et al 2007also reported that possible sitter for patients at risk for fall are highly recommended to prevent falls of patients with high risk of fall. Findings of this study showed also that the relationship between nurses working in general wards and their perception of risk factors towards patients' falls has higher significance than those nurses' participants from the critical care units. This findings may relate to the conditions of the patients' which is less mobility compared with patients in the general medical and surgical wards. Moreover, the nature of the critical nurses' job that has the ratio of nurse to patient as one to one. In other words, each nurse in critical care unit is working for one patient only all the shift, so ICU nurses are staying at close distance with their patients for close observation and giving care throughout the hour. This is fundamental to nursing care in ICU. This agrees with the findings of both (Chelly, et al. 2008). Meanwhile it is found that critical care nurses and general ward nurses have no significant difference in their effective interventions for preventing patients' falls. This agrees with (Tzeng 2015) that both nurses from critical units and general wards scored high mean in their response to the effectiveness of their interventions in preventing falls of patients on those units.

\section{Conclusion And Recommendation}

Fall prevention is an importance key practice toward patient falls. The more preventive measures considered in account, is this more risk factors identified. It is concluded that nurses' perception towards frequently perceived risk factors and their effective intervention to prevent those factors has positive strong relationship. Recommendation all nurses motivated and encouraged to attend regular continuous education every six months for risk falls and prevention measures. 


\section{Refrences}

[1] Oliver, D., Healey, F., \& Haines, T. P. (2010). Preventing fall and Fall-Related Injuries in Hospitals. Clinics in Geriatric Medicine, 26(4), 645-692. doi:10.1016/j.cger.2010.06.005

[2] Martin, F. C. (2011). Falls Risk Factors: Assessment and Management To Prevent Falls and Fractures. Canadian Journal on Aging / La Revue canadienne du vieillissement, 30(01), 33-44. doi:10.1017/s0714980810000747

[3] Macculloch, P. A., Gardner, T., \& Bonner, A. (2007). Comprehensive Fall Prevention Programs across Settings: A Review of the Literature. Geriatric Nursing, 28(5), 306-311. doi:10.1016/j.gerinurse.2007.03.001

[4] El Enein, N., El Ghany, A. and Zaghloul, A. (2012). Knowledge and performance among nurses before and after a training programme on patient falls. Open Journal of Nursing, 02(04), pp.358-364.

[5] Callis, N. (2016). Falls prevention: Identification of predictive fall risk factors. Applied Nursing Research, 29, pp.53-58.

[6] Eto, F. (2001). Causes of falls in the elderly. Japan Medical Association Journal, 44(7), 299305.

[7] Wayland, L., Holt, L., Sewell, S., Bird, J., \& Edelman, L. (2010). Reducing the Patient Fall Rate in a Rural Health System. Journal for Healthcare Quality, 32(2), 9-15. doi:10.1111/j.1945-1474.2009.00068.x

[8] Tung, E. E., \& Newman, J. S. (2014). Fall Prevention in Hospitalized Patients. Hospital Medicine Clinics, 3(2). doi:10.1016/j.ehmc.2013.11.005

[9] Al Jahdali H, Al Amoudi B, Abdulbagi D. (2009). Falls epidemiology at King Abdul Aziz University Hospital, Jeddah -Saudi Arabia- 2009. Life Science Journal, 9(2), 1174-8.

[10] Tzeng, H., \& Yin, C. (2013). Frequently Observed Risk Factors for Fall-Related Injuries and Effective Preventive Interventions. Journal of Nursing Care Quality, 28(2), 130-138. doi:10.1097/ncq.0b013e3182780037

[11] Hosein, A. FatollahIerad sh, HASSABI m, FalaknazI k, KhosravI SH, Poursaeed M. (2016). Fall Incidence and Risk Factors in Hemodialysis Patients. Turkish Nephrology, Dialysis and Transplantation Journal, 25 (3), 263-268.

[12] Ziere, G., Dieleman, J. P., Hofman, A., Pols, H. A., T. J. M. Van Der Cammen, \& Stricker, B. H. (2006). Polypharmacy and falls in the middle age and elderly population. British Journal of Clinical Pharmacology, 61(2), 218-223. doi:10.1111/j.13652125.2005.02543

[13] Mamun, K. and Lim, J. (2009). Association between falls and high-risk medication use in hospitalized Asian elderly patients. Geriatrics \& Gerontology International, 9(3), pp.276-281.

[14] Al Saif, A., Waly, E., \& Al Senany, S. (2015). The Prediction of Falls Among older people in Saudi Arabia. Journal of American Science, 8, 692-700

[15] Filler, K., Kelly, D. L., \& Lyon, D. (2011). Fall Risk in Adult Inpatients with Leukemia Undergoing Induction Chemotherapy. Clinical Journal of Oncology Nursing, 15(4), 369-370. doi:10.1188/11.cjon.369-370

[16] Al Senany, S. (2010). Prevention of patient falls in University Hospital. British Society of Gerontology.doi:http://www.britishgerontology.org/DB/gr-editions 2/generations-review/prevention-of-patient-falls-in-universityhospital.html.

[17] Williams, T., King, G., Hill, A., Rajagopal, M., Barnes, T., Basu, A., Pascoe, G., Birkett, K. and Kidd, H. (2007). Evaluation of a falls prevention programme in an acute tertiary care hospital. Journal of Clinical Nursing, 16(2), pp.316-324.

[18] Bunn, F., Dickinson, A., Simpson, C., Narayanan, V., Humphrey, D., Griffiths, C., Martin, W. and Victor, C. (2014). Preventing falls among older people with mental health problems: a systematic review. BMC Nursing, 13(1), 1-15, doi:10.1186/1472-6955-134.

[19] Gutierrez, F., \& Smith, K. (2008). Reducing falls in a definitive observation unit. Critical Care Nursing Quarterly, 31(2), 127-139.

[20] Chelly, J., Conroy, L., Miller, G., Elliott, M., Horne, J. and Hudson, M. (2008). Risk Factors and Injury Associated With Falls in Elderly Hospitalized Patients in a Community Hospital. Journal of Patient Safety, 4(3), pp.178-183.

Aisha Ramadan Alderby. "Nurses' Perception toward the Effectiveness of Using Risk Fall Prevention Protocol of Hospitalized Patients." IOSR Journal of Nursing and Health Science (IOSR-JNHS) 6.4 (2017): 09-14. 